\title{
Plastid genome evolution in the genus Allium
}

\author{
Victoria Scobeyeva \\ Lomonosov Moscow State University, \\ Moscow, Russia \\ Moscow Institute of Physics and \\ Technology, Moscow region, Russia \\ skobei-khanum@yandex.ru \\ Denis Omelchenko \\ Institute for Information Transmission \\ Problems, Moscow, Russia \\ omdeno@gmail.com \\ Maria Logacheva \\ Lomonosov Moscow State University, \\ Moscwo, Russia \\ Skolkovo Institute of Science and \\ Technology, Moscow, Russia \\ maria.log@gmail.com
}

\author{
Maxim Antipin \\ Lomonosov Moscow State University, \\ Moscow, Russia \\ sagefool@yandex.ru \\ Ilya Artyushin \\ Lomonosov Moscow State University, \\ Moscow, Russia \\ sometyx@gmail.com \\ Andrey Samoilov \\ Central Research Institute of \\ Epidemiology, Moscow, Russia \\ andrei.samoilov@gmail.com \\ Evgenii Konorov \\ Vavilov Institute of General Genetics \\ RAS, Moscow, Russia \\ crazydedula@mail.ru
}

\author{
Maxim Belenikin \\ Moscow Institute of Physics and \\ Technology, Moscow region, Russia \\ molecular.modeler@gmail.com \\ Anastasiya Krinitsina \\ Lomonosov Moscow State University, \\ Moscow, Russia \\ ankrina@gmail.com \\ Anna Speranskaya \\ Lomonosov Moscow State University, \\ Moscwo, Russia, Central Research \\ Institute of Epidemiology \\ Moscow, Russia \\ hanna.s.939@gmail.com
}

\begin{abstract}
Chloroplast genomes are highly conservative and plastomes of Alium specia are not an exception. We assembled and annotated plastomes of 12 specia from genus Allium. The only species with big inversion is A.paradoxa. We identified some regions of Allium plastomes that can possibly be under selection and some genes in these regions. The ycf1 gene is evolving at the highest rate.
\end{abstract}

\section{Keywords - Allium, plastome, evolution}

\section{Motivation}

Plastid genes are often used in plant molecular systematics and species identification due to their conservative sequences and high amount of copies in a single specimen. AtpF-H, matK, psbK-I, rbc ,ropC1,rpoB, trnH-psbA, and trnL-F were used most widely in past decade([1] and two of them - were selected as core plant barcodes. Nevertheless plastid genes code proteins, crucial for photosynthesis and should bear signs not only of neutral, but also of adaptive evolution.

\section{Aim}

Specia of Alium genera inhabit wide range of environments with various insolation, humidity, average temperature and other parameters, affecting photosynthesis. We sequenced and assembled plastid genomes of 12 Alium specia and tried to find signatures of natural selection in plastid genes.

\section{Methods}

We sequenced and assembled de novo plastomes of the following species: A. fistulosum L., A. macleanii Baker, A. moly L., A. nutans L., A. obliquum L., A. platyspathum Schrenk, A. pskemense B.Fedtsch., A. schoenoprasum L., Allium semenovii Regel, Allium tuberosum Rottl. ex Spreng., A. victorialis L., Allium zebdanense Boiss. \& Noë. cpDNA was extracted from living specimen with standard CTAB protocol. DNA samples were sequenced using the Illumina MiSeq high-throughput sequencing platform. The TruSeq protocol (NEBNext ${ }^{\circledR}$ DNA Library Prep Master Mix Set for Illumina, E6040, NEB reagents) was used for preparing the genomic libraries according to manufacturer's recommendation. PE sequences $(2 \times 250 b p$ or $2 \times 300 b p)$ using
MiSeq. After the quality trimming with Trimmomatic sequencing reads were filtered using chloroplast genome sequences of A. cepa and A. sativum by Bowtie mapper. Dual Organellar GenoMe Annotator (DOGMA) and GeSeq were used for gene annotation. Alignments for evolution rate analysis of each region of chloroplast genome were built using MUSCLE. FUBAR was used for selection seeking. Phylogenetic trees were made with iqtree, using nucleotide alignment. With iqtree Partition finder were determined 3 partitions and evolution rates were calculated independently for each partition.

\section{Results}

We have compared the gene order between plastomes of Allium species sequenced de novo and of species sequenced previously The general structure and gene order across all species show high similarity and absence of rearrangements, with A. paradoxum, being the only exception. This species has a major inversion described in our previous work We have not found any large inversions in any other studied species of Allium [2]. So we conclude that plastome of A. paradoxum has a very unusual structure for the genus. Several regions under selection within Allium plastomes were identified. The major region is located from ycfl (the last gene of the IRa) to ycf2 (the first gene of IRb) and covers the whole SSC region (except only trnL). Partitions evolve with quite different speed from $0.16 \quad$ (genes clpP rpl2 rps7 rps12 rpl2) to 1.43 (genes psbM psbL ndhD ndhE ndhI ndhA). Alium plastomes are highly conservative. Alignment has 13 sequences with 45030 columns, 614 distinct patterns, 879 parsimony-informative and 1410 singleton sites.

\section{Acknowledgment}

Supported by the RFBR (18-04-01203).

\section{References}

[1] Dong et al. (2015) Ycf1, the most promising plastid DNA barcode of land plants. Scientific reports. $5: 8348$

[2] Omelchenko et al. (2019) Complete plastome sequencing of Allium paradoxum reveals unusual rearrangements and the loss of the ndh genes as compared to Allium ursinum and other onions. Gene:144-154 